\title{
Morphologie des valves et évolution du cingulum chez Achnanthes inflata (Bacillariophyceae)
}

\author{
R. Le Cohul
}

Mots clès : Bacillariophycées, Achranthes, frustule, valve, cingulum.

Achanthes inflata (Kütz) Grun. et la variété elata (Leud.Fontm.) Hust. ont été étudiés au microscope électronique. L'espèce et la variété, outre la forme de la valve, se différencient par quelques caractères structuraux. Une attention particulière a été portée sur l'organisation du cingulum ; le nombre d'éléments qui le composent est variable en fonction du stade où se trouve la cellule entre deux divisions.

The morphology of valves and evolution of the cingulum in Achnanthes inflata (Bacillariophyceae).

Keywords : Bacillariophyceae, Achnanthes, frustule, valve, cingulum.

Achnanthes inflata (Kütz.) Grun. and the variety A. inflata var. elata (Leud.-Fontm.) Hust. were examined using electron microscopy. The species and the variety, besides the valve shape, present a few dissimilarities in the structural characters. A particular attention was paid to the girdle region; the cingulum is composed of a variable number of bands depending on the stage in the cycle life of the cell,

\section{Introduction}

Achnanthes inflata (Kütz.) Grun. a une très large répartition géographique (Frenguelli 1941, Hustedt 1959, Gandhi 1960 et 1966, Patrick \& Reimer 1966, Foged 1971, 1976, 1978, 1979 et 1983, Germain 1981, Cassie 1981, Carter \& Bailey-Watts 1981). En Afrique, cette espèce a déjà été signalée par Cholnoky (1962 a et b, 1963, 1966 et 1968), Schoeman (1973) et Gasse (1986), et sans doute cet te liste n'est-elle pas exhaustive. Cette étude a été réalisée à partir d'un prélèvement en provenance du Maroc (source Chella) où ce taxon n'avait jamais été signalé jusqu'à ce jour.

La variété elata (Leud.-Fontm.) Hust. a été décrite par Leuduger-Fontmorel (1878, in Patrick \& Reimer 1966) dans l'île de Ceylan. Ce taxon semble avoir une distribution géographique voisine de l'espèce bien qu'il soit plus rarement signalé : Hustedt

\footnotetext{
1. Laboratoire d'Hydrobjologie, URA 0695 du C.N.R.S., Université Paul Sabatier, 118 route de Narbonne, 31062 Toulouse Cédex, France
}

(1937-1938), Frenguelli (1941), Gandhi (1960 et 1966) qui en fit une espèce à part entière sous le nom d'A. elata (Leud.Fontm.) Gandhi, Foged (1979), Germain (1981) et Le Cohu \& Maillard (1983). Dans cette étude, seuls seront apportés, par rapport à la description des deux derniers auteurs cités, quelques compléments d'information ; les spécimens étudiés proviennent des îles Kerguelen (eau douce).

\section{Méthodes}

Les frustules ont d'abord été nettoyés, soit à l'acide sulfurique, soit à l'eau oxygénée (110 volumes). Par la suite, les échantillons ont été traités de manière classique suivant les nécessités de l'observation, c'est-à-dire soit montés dans une résine synthétique (naphrax), soit métallisés à l'orpalladium (les frustules préalablement séchés sur des lamelles de verre étaient fixés sur les porteobjets à l'aide de vernis à ongles), soit posés sur des grilles de collodion carboné. (Microscope photonique M.P., microscope électronique à balayage M.E.B., microscope électronique à transmission M.E.T.). 
La sonication a été également utilisée pour essayer de briser les frustules ou de séparer les différents éléments de la ceinture.

La terminologie utilisée est généralement celle de Ross et al. (1979); les changements sont indiqués dans le texte.

\section{Résultats}

\section{Achnanthes inflata (Külz.) Grun.}

Au microscope photonique (fig. 1 et 2), A. inflata se présente sous sa forme typique avec 3 renflements (fig. 1 et 2). Au M.E.B., la valve à raphé est concave (fig. 4); en vue externe, les fissures terminales du raphé s'infléchissent presque perpendiculairement à l'aire axiale ( $f \mathrm{ig} .3$ et 9 ) et se poursuivent lon. guement sur le manteau. En vue inteme, comme chez la var. elata (Le Cohu \& Maillard 1983), les 2 branches du raphé se terminent en crochet au niveau de l'aire centrale (fig. 6) ; celle-ci est marquée par un stauros (fig. 4, 5,6) toujours demuni d'intercôtes (Krammer 1982); aire centrale et aire axiale sont fortement silicifiées ( $/$ ig. 5). Les parois valvaires (fig. 12 et 18) sont apparemment constituées de 2 couches siliceuses et seraient donc de type loculaire (Ricard 1987). La suture (fig. 4, 10 et 11), assez large, est ornementée d'excroissances siliceuses (blisters, Rounds 1984) dont l'origine et la signification sont inconnues jusqu'à ce jour. La valve sans raphé est légèrement bombée au niveau de "l'aire centrale "(fig. 11); elle se caractérise par la présence d'un aileron marginal ( $\mathrm{fig} .4$ et 11) plus ou moins fragile qui interrompt les côtes et les intercôtes (Krammer 1982); la présence d'une bordure périphérique, mais dentelée, a déjà été mise en évidence chez $A c h$ nanthes lanceolata (Bréb.) Grun. var. lanceolatoides (Sov.) Reim. (Le Cohu \& Maillard 1983) et A. marginulata Grun. (Lange-Bertalot \& Le Cohu 1985). L'aire axiale est complètement déjetée sur le côté (fig. 7 et 23), à la limite du manteau. Comme sur la valve à raphé, la suture est ornementée d'excroissances siliceuses ( $f$ g. 4 ).

Les côtes et les intercôtes (Krammer 1982) entourent complètement les 2 valves. Les intercôtes sont constituées de gandes aréoles plus ou moins quadrangulaires $(1 \# 0,4 \mu \mathrm{m})$. Au M.E.B., l'organisation de ces dernières est souvent difficile à me ttre en évidence ; elle se caractérise par la présence à l'extérieur d'un velum (fig. 9) déjà figuré par Okuno (1974) au M.E.T. ; ces velums sont constitués d'un ensem. ble de volae ou, plus précisément, de ce que Mann (1981) appelle " volae portées sur un crib rum " (cribum borne-volae). Ce type d'occlusion du lumen se retrouve chez d'autres Achanthes du même groupe, tels $A$. brevipes (Helmcke \& Krieger 1962), A. brevipes var intermedia (Okuno 1970), A. crenulata (Okuno 1974), A. kurvaitensis (Mann 1981), A. st. paulii (Heid. et Kolbe) Kobay. et Sawat. (Kobayasi \& Sawatari 1986). Aucune vue interne de ces velums n'a pu être observée.

Pour toute étude du cingulum, il faut considérer que toute cellule de diatomée représente un état transitoire, souvent relativement bref, entre deux divisions: dans la nature, notamment quand la population est peu nombreuse, il est difficile de discerner la phase mature. La composition du cingulum (Crawford 1981) évolue en fonction du moment où l'on effectue l'observation : phase de division ou non. Tout d'abord, il faut remarquer que, contrairement à ce qui est parfois affirmé (Gerrnain 1981 . Ricard 1987), la valve à raphé peut être épivalve, ce qui semble logique étant donné le mode de division des diatomées. Les figures 10 et 11 illustrent ce propos. La première montre une valve sans raphé néoformée, encore recouverte en partie par deux bandes cingulaires $\mathrm{Cl}$ et $\mathrm{C} 2$, la dernière étant difficilement visible: la figure 11 représente un stade où l'hypocingulum commence à se développer; il faut

Planche 1. Achnanthes inflata. Fig. 1 : valve à raphé. M.P. ; Fig. 2 : valve sans raphé. M.P. ; Fig. 3 : valve à raphé, vue externe ; Fig. 4 : vue externe d'un frustule entier ; excroissances siliceuses (flèche); Fig. 5 : valve à raphé, vue interne de la face valvaire et vue externe du manteau; Fig. 6 : détail du stauros montrant les terminaisons en crochet des 2 branches du raphé sur la face interne; Fig. 7 : valve sans raphé ; aire axiale en vue interne déjetée contre le manteau visible également en vue externe; présence de la valvocopula (flèche); Fig. 8 : détail de la figure 7 mont rant les indentations de la valvocopula s'insérant sur les côtes de la valve, décrochement (flèche étêtée); Fig. $9:$ vue externe avec fissure terminale du raphé et structure des aréoles ; Fig. 10 : frustule entier ; fissure terminale du raphé (flèche noire) ; excroissances siliceuses (flèche noire étêtée), valvocopula (V.); bandes cingulaires (C1 et C2); ligule (flèche blanche); Fig. 11 : frustule entier, ligule (flèche); Fig. 12 : vue externe de valve à raphé brisée, 2 couches de silice (flèches noire et blanche). Fig. 1, 2, 4, 5 et 11 (trait central): échelle $10 \mu \mathrm{m}$. 

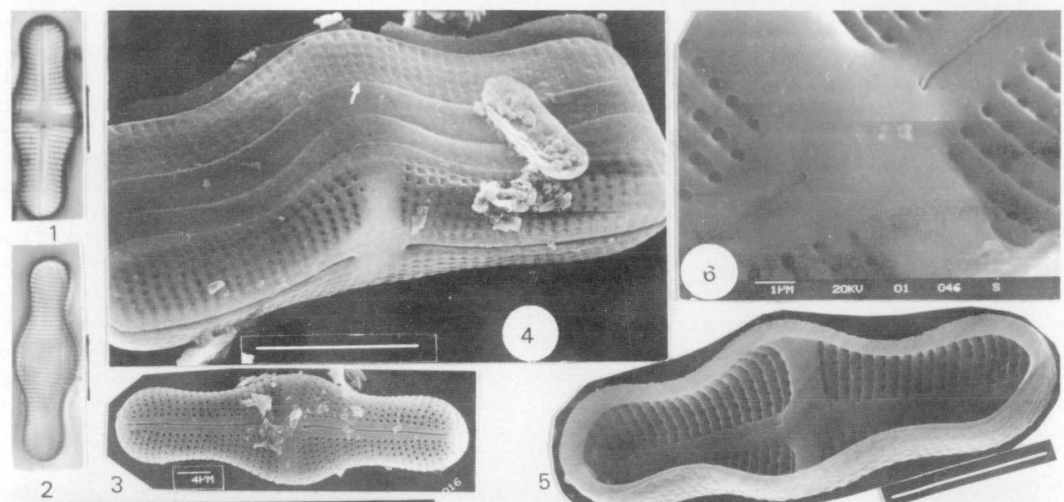

4
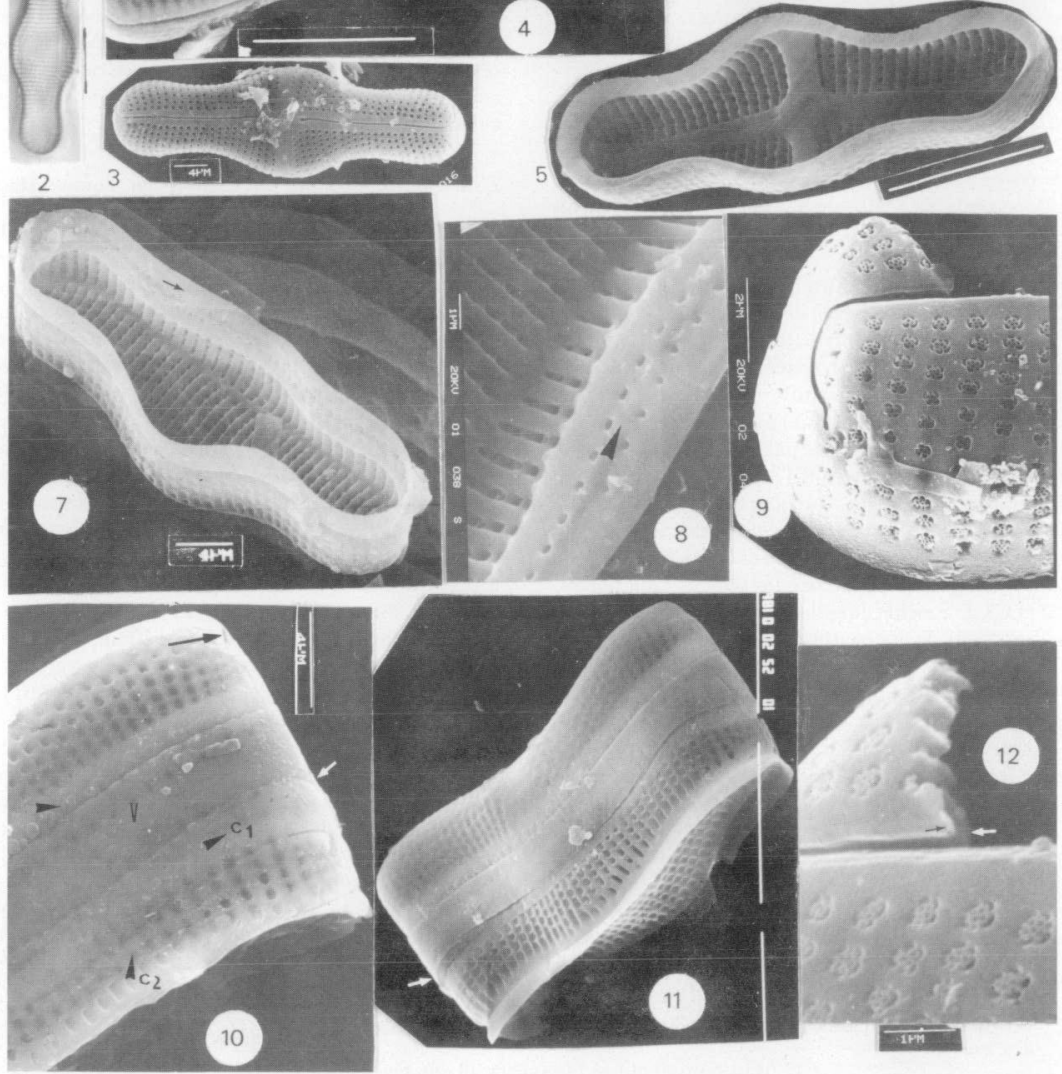
noter la présence d'un bec (flèche blanche) assimilable à une ligule (cf. aussi fig. 10) sur la valcopula de l'épicingulum. La figure 13 (cf. aussi fig. 4) se rapporte à un autre stade de développement du frustule; les deux valves sont séparées par deux bandes cingulaires, de largeur sensiblement équivalente, que l'on peut assimiler à deux valvocopulas. Ce stade peut être interprété comme le stade mature du frustule. Ces valcocopulas sont pourvues d'indentations (fig. 16 et 17) qui s'imbriquent sur les côtes de la valve ( $f$ ig. 8), système analogue à celui observé chez les Synedra et les Fragilaria (Williams 1986, Le Cohu 1988) et chez certaines Navicula (Phips \& al. 1983) ; elles sont ornées de 2 rangées de pores, surtout visibles en vue interne ( $f$ ig. 8,16 et 24); ces pores ont une structure de type "volae " (fig. 25) analogue à celui des aréoles. Au niveau de la rangée advalvaire de pores, se situe un décrochement permettant à la valcocopula de s'insérer à l'intérieur de la valve (fig. 8, 16 et 17).

La figure 14 illustre une autre phase du développement du cingulum avec des cellules qui viennent de se diviser et dont 3 frustules restent encore liés ensemble (fig. 15). Si l'on observe la figure 14 de bas en haut, le premier frustule montre une valve à raphé néoformé (V2) qui résulte de la division de l'ancien frustule constitué par les valves V1 et V4 ; 3 éléments du cingulum (C1, C3 et $\mathrm{C} 4)$ qui réunissaient ces deux valves recouvrent encore V2. La valve à raphé V4 est devenue l'épivalve de la valve sans raphé néoformée V3, celle-ci étant encore enveloppée en grande partie par les bandes cingulaires $\mathrm{C} 2$ et $\mathrm{C} 5$ de l'ancien cingulum entre V1 et V4. Ce cingulum était donc constitué de 5 éléments, C1, C2, $\mathrm{C} 3, \mathrm{C} 4$ et $\mathrm{C} 5$, tous munis de "ligules " plus ou moins longues; $\mathrm{C} 1$ et $\mathrm{C} 2$ peuvent être assimilés aux valvocopulas. Si l'on admel que la seule présence de 2 valvocopulas représente le stade "mature" du frustule, les bandes cingulaires $\mathrm{C} 3, \mathrm{C} 4$ et $\mathrm{C} 5$ constituent une zone d'élargissement de l'espace intervalvaire analogue à la zone d'élongation observée, par exemple, chez les Melosira au moment de la division cellulaire. Chez A. inflata, cette zone doit se former également pendant cette phase de la vie de la cellule et a un rôle de protection pendant la formation des nouvelles valves. L'aileron marginal (a) de la valve sans raphé $\mathrm{V} 5$ réunit cette dernière à $\mathrm{V} 4$. V5 et V8 faisaient partie du même frustule, mais on peut déceler les 2 valves, V6 et V7, néoformées sous le cingulum ; celui-ci, comme le précédent $(\mathrm{V} 1, \mathrm{~V} 4)$ est constitué de 5 bandes cingulaires $\left(C^{\prime} 1, C^{\prime} 2, C^{\prime} 3\right.$, C'4 et C'5). Les indentations visibles sur C'5 posent un problème d'interprétation ; leur présence laisse supposer que les copulas en sont pourvues; nos observations ne nous permettent pas de déterminer comment ces indentations s'insèrent sur les valvocopulas.

\section{Achnanthes inflata var, elata (Leud,-Fontm.) Hust}

Une première description, incomplète, de $A$. inflata var. elata au M.E.B. a déjà été donnée (Le Cohu \& Maillard 1983). Outre la forme, ce qui distingue la variété de l'espèce, c'est la présence d'intercôtes au niveau de l'aire centrale sur la valve à raphé (fig. 20) et celle d'un orbiculus (Mc Intire \& Reimer 1974) aux extrémités de la valve sans raphé (fig. 21). Cet orbiculus a été assimilé à un nodule terminal chez A. temperei par Patrick \& Reimer (1966) et à une aréole par Hendey (1951); cette dernière interprétation est également celle de Kobayasi et Sawatari (1986). Des observations effectuées au M.E.T. confirment cette version; l'orbiculus est une aréole différente des autres par la forme et la taille (fig. 27 et 28); il est fermé par un velum qui semble de type cribrum, Il est toutefois difficile de discerner si on a affaire à une vue interne ou externe, la première étant la plus vraisemblable; en effet, la figure $\mathbf{2 6}$ représente une vue externe des aréoles avec une structure de l'occlusion du lumen tout à fait comparable à celle rencontrée chez l'espèce type. Si on compare cette figure 26 avec une vue interne (Fig. 22, difficile à distinguer mais visible)

Planche 2. Achnanthes inflata. Fig. 13 : frustule entier avec 2 valvocupulas; Fig. 14 : frustules venant de se diviser (explications dans le texte); Fig. 15 : détail de la figure 14 à un plus fort grossissement et inclinée à $45^{\circ}$ montrant la série V3 - V8; Fig. 16 : valvocopula ou copula (cf. texte), décrochement (flèche) ; Fig. 17 : valvocopula (copula), vue externe, ligne de pores renforcée artificiellement; Fig. 18 : fraction de valve à raphé montrant les 2 couches de silice (flèches). Achnanthes inflata var. elata. Fig. 19 : vue interne de la valve à raphé et présence de 2 bandes cingulaires; Fig. 20 : valve à raphé, vue interne : Fig. 21 : valve sans raphé, vue externe, orbiculus (flèches); Fig. 22 : valve à raphé, vue interne des aréoles.

Fig. 13 (trait central) et Fig. 17 : échelle $10 \mu \mathrm{m}$. Fig. 22 : échelle $1 \mu \mathrm{m}$. 


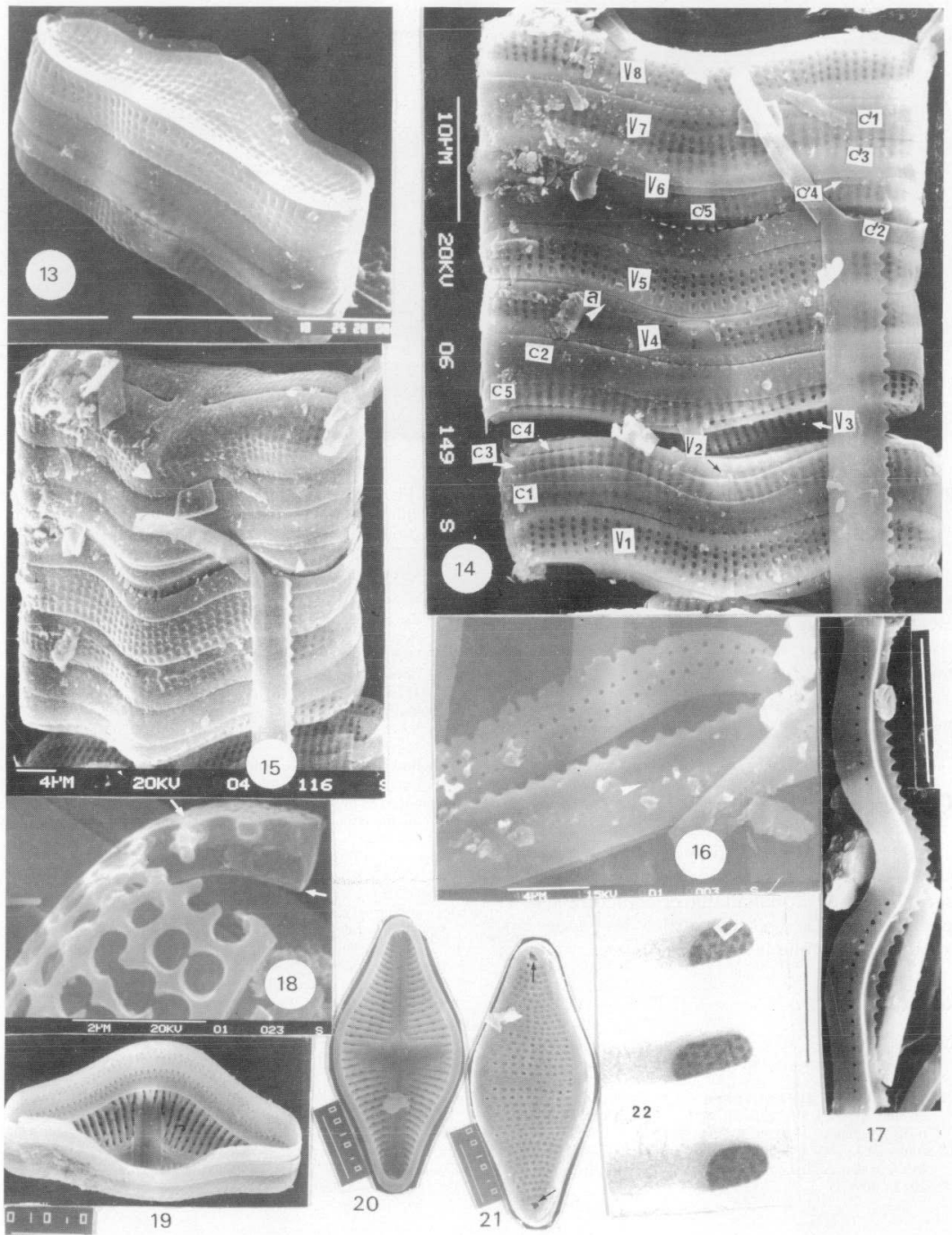



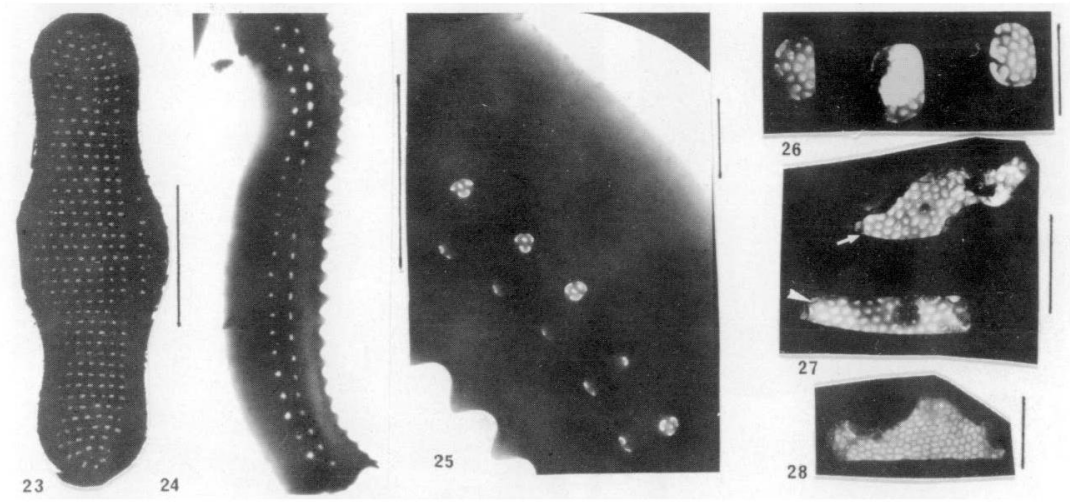

Planche 3. (M.E.T.). Achnanthes inflata. Fig. 23 : valve sans raphé ; Fig. 24 : valvocopula (ou copula); Fig. 25 : détail des aréoles de valcocopula (ou copula), vue externe. Achnanthes inflata var, elata. Fig. 26 : détail des aréoles, vue externe : Fig. 27 : orbiculus (flèche), aréole (flèche étêtée), sans doute vue interne; Fig. 28 : orbiculus, vue interne? Fig. 23 et 24 : échelle $10 \mu \mathrm{m}$. Fig. $25.26,27$ et 28 : échelle $1 \mu \mathrm{m}$.

et si l'on admet que la grande aréole (fig. 27, flèche étêtée) est une vue inteme, la dénomination de Mann (1981) " volae portées sur un cribrum " est parfaitement justifiée.

Une seule observation du cingulum a pu être réalisée : comme chez l'espèce type, les bandes intercalaires possèdent 2 rangées de pores (fig. 19).

\section{Conclusion}

Il est évident que cette étude a besoin d'être pour. suivie, aussi bien sur l'espèce que sur la variété. Il serait notamment souhai table de connaître la morphologie fine et le mode d'insertion des bandes cingulaires autres que les valvocopulas.

\section{Travaux ciles}

Carter (J.R.) \& Bailey-Watts (A.E.). 1981, - A taxonomic study of diatoms from standing freswater in Shet land. Nova Hedwigia, $3.3: 513-630$

Cassie (V.). 1981. - The fossil and living freshwater diatom flora of New-Zealand. Proceed. 6th Symp. Recent and Fossil Diatorns, Budapest, 1980. O. Koeltz: 321-338.

Cholnoky (B.J.). 1962 a. - Ein Beitrag zu der Okologie der Diato meen in dem englishen Protektorat Swaziland. Hydrobiologia, $20,4: 309.355$
Cholnoky (B.J.). 1962 b. - Beiträge zur Kenntnis der Südafrikanischen Diatom een flora. III. Diatomeen aus der Kaap-Provinz. Revista Biol., 3. 1: 1-80.

Cholnoky (B.J.). 1963. - Ein Beitrag zur Kenntnis der Diatomeenflora from Hollandisch-Neuginea. Nova Hedwigia, 5, 1/2: 157-201.

Cholnoky (B.J.). 1966. - Diatomeenassoziationen aus einigen Quellen in Sïdwest-Afrika und Bechuanaland. Nova Hedwigia, 21 $163-249$.

Cholnoky (B.J.). 1968. - Die Diatomeenassoziationen der SantaLucia Lagune in Natal. Butanica marina, suppl., XI : 1.227.

Crawford (R.M.) . 1981. - Valve formation in diatoms and the fate of the silicalemna and plasmalemma. Protoplasma, 106: 157-166.

Foged (N.). 1971. - Freshwater diatoms in Thailand. Nova Hedwigia, 22, $1 / 2: 267-368$.

Foged (N.). 1976. - Freshwater diatoms in Sri Lanka (Ceylan). Bibliotheca phycol., 23 : 113 pages.

Foged (N.). 1978. - Diatoms in Eastearn Australia. Bibliotheca Phycol, $41: 243$ pages.

Foged (N.). 1979. - Diatoms in New-Zealand, the North Island. Bibliotheca Phycol., 47 : 225 pages.

Foged (N.). 1983. - Diatoms in fountains, reservoirs and some other humid and dry localities in Rome, Italy. Nova Hedwigia, $38: 433-469$

Frenguelli (J.). 1941, - Diatoneas del rio de la Plata. Rev del Museo de la Plata, N.S., Sec. Bot., III, $15: 213-334$.

Gandhi (H.P.). 1960. - Some new diatoms from the Jog-Falls (Mysore Siate), J.R. Micr. Soc., 79, 1:81-87.

Gandhi (H.P.). 1966. - The freshwater diatomfiora of the Jog-Falls, Mysore State. Nova Hedwigia, 11, 1-4; 89.197, 9 pl.

Gasse (F.). 1986. - East African diatoms. Taxonomy, ecological distribution. Bibliotheca Diatomologica, 11 : $245 \mathrm{p}$. 
Germain (H.). 1981. - Flore des diatomées, eaux douces et saumâtres. Boubée, Paris : 444 p.

Helmcke (J.G.) \& Krieger (W.). 1962. - Diatomeenschalen in elektronenmikroskopischen Bild, 1, J. Cramer, Weinheim : pl. 49.

Hendey (N.1.). 1951 - - Littoral diatoms of Chichester Harbour with special reference to fouling, J. Roy. Mic, Soc, ser, 3, $71 ; 1-86$.

Hustedt (F.). 1937/1938. - Systematische und Okologische Untersuchungen über die Diatomeenflora von Java, Bali und Sumat ra-Systematischer Teil. Arch. Hydrobiol., Suppl. 15/16: $131.177 ; 187-295 ; 393.506$

Hustedı \{F, \}. 1959. - Die Kieselagen. In : Kryptogamen-Flora von Deutschland. Österreich und der Schweiz. Bd VII, Part 2, Rabenhorst, Leipzig : 845 p.

Kobayasi (H.) \& Sawatari(A.). 1986. - Some small and elliptic Achnanthes. 8 th. Diatom Symposium 1984 : 259-269.

Krammer (K.). 1982. - Observations on the alveoli and aerolae of some Naviculaceae. Nova Hedwigia, 73 ; 55-79.

Lange-Bertalot (H.) \& Le Cohu (R.). 1985. - Raphe like vestiges in the pennate diatom suborder Araphjdineae? Annls Limrol, 21. $3: 213.220$

Le Cohu (R.). 1988 - Fragilaria alpestris, Opephora naveara nov. sp. et le complexe Synedra ulna (Bacillariophycées, Araphidinées) : Morphologie et ultrastructure. Cryptogamie. Algologie, $9,2: 101.116$

Le Cohu (R.) \& Maillard (R.). 1983. - Les diatomées monoraphidées des iles Kerguelen. Annls Limnol., 21, 3: 143.167.

Mc Intire (C.D.) \& Reimer (C.W.). 1974. - Some marine and brackish-water Achnanthes from Yaquina Estuary, Oregon (U.S.A.). Botanica marina, $17: 164-175$.
Mann (D.G.) 1981 . - Sieves and flaps : Siliceous minutiae in the pores of raphid diatoms. Proceed 6th Symp. Recent and Fossil Diatoms. Budapest 1980, O. Koeltz: 279-300.

Okuno (H.). 1970. - Marine diatorns. In Helmcke J.G. et W. Krieger (ed), Diatomeenschalen im electronenmikroskopischen Bild, 7, Cramer. Lehre: pl. 679-683.

Okuno (H.). 1974. - Freshwater diatoms. In Helmcke I.G. et W. Krieger (ed.), lbid., 9: pl. 846-850, 857 et 858.

Patrick (R.) \& Reimer (C.W.). 1966. - The Diatoms of the United States exclusive of Alaska and Hawai. Vol. I. Acad. Nat. Sc. Phi. ladelphia, Monog. 13: 688 p.

Phips (D.W.), Rosowski (J.R.) \& Rosowski (J.) 1983. - The morphology and integration of valves and bands in Navicula mutica var. mutica (Bacillariophyceae). J. Phycol, $19: 320-333$.

Ricard (M.). 1987. - Atlas du phytoplancton marin. Vol. 2 : Diatomophycées. Ed. C.N.R.S. Paris : 297 p.

Russ (R.), Cox (E.J.), Karajeva (N.I.), Mann (D.G.), Paddock (T.B.B.), Simonsen (R.) \& Sims (P.A.). 1979. - An amended terminology for the siliceous components of the diaton cell. Nova Hedwi. gia, 64 : $512-533$.

Round (F.E.). 1984. - The circumscription of Synedra and Fragilaria and their subgroupings. 7th. Diatom Symposium 1982, Koeltz, Koeningstein : 241-253.

Schoeman (F.R.). 1973. - A systematical and ecological study of the diatom flora of Lesotho with special reference to the water quality. V. et R. Printers, Pretoria : $366 \mathrm{p}$.

Williams (D.M.). 1986. - Comparative morphology of some species of Synedra Ehrenb. with a new definition of the genus. Dia tom Research, 1, $1: 131.152$. 